Pacific Journal of Mathematic 


\title{
THE DIMENSION OF THE KERNEL OF A PLANAR SET
}

\author{
MARILYN BREEN
}

Let $S$ be a compact subset of $R^{2}$. We establish the following: For $1 \leqq k \leqq 2$, the dimension of $\operatorname{ker} S$ is at least $k$ if and only if for some $\varepsilon>0$, every $f(k)$ points of $S$ see via $S$ a common $k$-dimensional neighborhood having radius $\varepsilon$, where $f(1)=4$ and $f(2)=3$. The number $f(k)$ in the theorem is best possible.

We begin with some definitions: Let $S$ be a subset of $R^{d}$. For points $x$ and $y$ in $S$, we say $x$ sees $y$ via $S$ if the segment $[x, y]$ lies in $S$. The set $S$ is starshaped if there is some point $p$ in $S$ such that, for every $x$ in $S, p$ sees $x$ via $S$. The set of all such points $p$ is called the (convex) kernel of $S$, denoted by $\operatorname{ker} S$.

A well-known theorem of Krasnosel'skii [5] states that if $S$ is a compact set in $R^{d}$, then $S$ is starshaped if and only if every $d+1$ points of $S$ see a common point via $S$.

Although various results have been obtained concerning the dimension of the set $\operatorname{ker} S$ (Hare and Kenelly [3], Toranzos [6], Foland and Marr [2], Breen [1]), it still remains to set forth an appropriate analogue of the Krasnosel'skii theorem for sets whose kernel is at least $k$-dimensional, $1 \leqq k \leqq d$. Hence the purpose of this work is to investigate such an analogue for subsets of the plane.

The following terminology will be used. Throughout the paper, conv $S, \operatorname{cl} S$, int $S$, bdry $S$, and ker $S$ denote the convex hull, closure, interior, boundary, and kernel, respectively, of the set $S$. If $S$ is convex, $\operatorname{dim} S$ represents the dimension of $S$. Finally for $x \neq y, R(x, y)$ denotes the ray emanating from $x$ through $y$ and $L(x, y)$ is the line determined by $x$ and $y$.

2. The results. We begin with the following theorem for sets whose kernel is 1-dimensional.

TheOREM 1. Let $S$ be a compact set in $R^{2}$. The dimension of ker $S$ is at least 1 if and only if for some $\varepsilon>0$, every 4 points of $S$ see via $S$ a common segment of radius $\varepsilon$. The number 4 is best possible.

Proof. The necessity of the condition is obvious. Hence we need only establish its sufficiency. 
By Krasnosel'skii's theorem in $R^{2}, S$ is starshaped, so we may select a point $z$ in ker $S$. Moreover, we assert that every 4 points of $S$ see a common segment of length $\varepsilon$ having $z$ as endpoint (we refer to such a segment as an $\varepsilon$-interval at $z$ ): For $x_{1}, x_{2}, x_{3}, x_{4}$ in $S$, these points see a common $2 \varepsilon$-interval $[a, b]$ in $S$, and since $z \epsilon$ ker $S, \operatorname{conv}\left\{z, x_{i}, a, b\right\} \subseteq S$ for each $1 \leqq i \leqq 4$. Hence $x_{\imath}$ sees $\operatorname{conv}\{z, a, b\}$ for every $i$. Certainly one of the edges $[z, a],[z, b]$ of the triangle (possibly degenerate) $\operatorname{conv}\{z, a, b\}$ has length at least $\varepsilon$, and this edge satisfies our assertion.

To complete the proof, we consider two cases.

Case 1. Assume that $z \in$ int $S$. Let $N$ be a disk about $z$ of radius $r \leqq \varepsilon$ contained in $S$. If $N=S$ the result is immediate, so assume that $S \sim N \neq \dot{\phi}$. For $y \in S \sim N$, we define $C_{y}$ to be the subset of $N$ seen by $y$. Since $S$ is starshaped, $S$ is simply connected, so $C_{y}$ is convex. Let $\left[a_{y}, b_{y}\right]$ be the intersection of $C_{y}$ with the line perpendicular to $L(y, z)$ at $z$, and let $\delta_{y}$ be the smaller of the lengths of the segments $\left[a_{y}, z\right]$ and $\left[b_{y}, z\right]$, say the length of $\left[a_{y}, z\right]$.

If glb $\delta_{y}>0$, then $\cap C_{y}$ contains a neighborhood of $z$, contained in ker $S$. Hence we may assume glb $\delta_{y}=0$.

Let $\left\{y_{n}\right\}$ be a sequence of points in $S$ such that $\delta_{y_{n}} \rightarrow 0$ as $n \rightarrow \infty$. Let $y_{0}$ be a limit point of $\left\{y_{n}\right\}$ and assume $y_{n}$ converges to $y_{0}$. Set $L=L\left(y_{0}, z\right)$ and call the open halfplanes into which $L$ divides the plane $L_{1}$ and $L_{2}$. Without loss of generality, we assume that for each $n$, the corresponding $a_{n}$ lies in the closed halfplane cl $L_{2}$ determined by $L$.

We now show that every two points of $S$ see a common $\varepsilon$-interval at $\approx$ in $\operatorname{cl} L_{1}$ : Otherwise, some members $x_{1}$ and $x_{2}$ of $S$ would see no such interval, and there would exist points $q_{1}$ and $q_{2}$ in bdry $N \cap L_{2}$ such that every $\varepsilon$-interval at $z$ seen by both $x_{1}$ and $x_{2}$ would lie in the convex region bounded by rays $R\left(z, q_{1}\right)$ and $R\left(z, q_{2}\right)$. However, for $\delta_{n}$ sufficiently small, $y_{n}$ sees no $\varepsilon$-interval at $z$ in this region, impossible since $x_{1}, x_{2}, y_{n}$ see a common $\varepsilon$-interval at $z$. Thus the result is established.

Assume that the points of bdry $N \cap \mathrm{cl} L_{1}$ are ordered in a clockwise direction from $s_{0}$ to $t_{0}$, where $s_{0}$ and $t_{0}$ denote the endpoints of the interval $N \cap L$. For each $y$ in $S$, there exist $s_{y}$ and $t_{y}$ on bdry $N \cap$ cl $L_{1}$ such that $y$ sees $\left[s_{y}, z\right] \cup\left[t_{y}, z\right]$ via $S$ and such that $s_{y}$ and $t_{y}$ are, respectively, the first and last points on bdry $N \cap \mathrm{cl} L$ having this property. Finally, let $E_{y}$ denote the convex hull of all segments 
$\left[z, a_{y}\right]$ seen by $y$, where $a_{y} \in \operatorname{bdry} N \cap \operatorname{cl} L_{1}$. Certainly $y$ sees $E_{y}$ via $S$.

We say $a<b$ on bdry $N \cap \operatorname{cl} L_{1}$ if $a$ precedes $b$ in our clockwise order. Since every pair of points of $S$ sees a common $\varepsilon$-interval at $z$ in $\mathrm{cl} L_{1}$, it follows that lub $s_{y} \leqq$ glb $t_{y}$. Let $s_{1}=\operatorname{lub} s_{y}$ and $t_{1}=$ glb $t_{y}$. Then for each $y$ we have $s_{0} \leqq s_{y} \leqq s_{1} \leqq t_{1} \leqq t_{y} \leqq t_{0}$. If $s_{0}=s_{1}$ or $t_{1}=t_{0}$, the proof is complete. Hence we assume that $s_{0} \neq s_{1}$ and $t_{1} \neq t_{0}$, so that conv $\left\{s_{1}, z, t_{1}\right\} \cap L=\{z\}$. If for some positive number $r^{\prime}$, the set $\cap E_{y}$ contains an interval of length $r^{\prime}$ in $\operatorname{conv}\left\{s_{1}, z, t_{1}\right\}$, the proof is finished. Otherwise, for every $1 / n$ there is some $w_{n}$ in $S$ for which $E_{w_{n}}=E_{n}$ does not contain $M(z, 1 / n) \cap \operatorname{conv}\left\{s_{1}, z, t_{1}\right\}$, where $M(z, 1 / n)$ denotes the $1 / n$-disk centered at $z$. Hence the sequence of sets $E_{n}$ converges to $\left[s_{0}, t_{0}\right]$.

In this case, every point of $S$ sees some $\varepsilon$-interval at $z$ on $L$ : Suppose on the contrary that for some $x$ in $S, x$ sees neither $\left[s_{0}, z\right]$ nor $\left[z, t_{0}\right]$ via $S$. Then there exist points $p_{1}$ and $p_{2}$ in bdry $N \cap L_{1}$ and points $p_{1}^{\prime}$ and $p_{2}^{\prime}$ in bdry $N \cap L_{2}$ such that every $\varepsilon$-interval at $z$ seen by $x$ lies either in the convex region bounded by $R\left(z, p_{1}\right) \cup$ $R\left(z, p_{2}\right)$ or in the convex region bounded by $R\left(z, p_{1}^{\prime}\right) \cup R\left(z, p_{2}^{\prime}\right)$. However, for $n$ sufficiently large, the points $y_{n}$ and $w_{n}$ defined previously see no common $\varepsilon$-interval at $z$ in either of these regions, impossible since every 4 points of $S$ see a common $\varepsilon$-interval at $z$. Thus the assertion is proved.

Finally, we have to show that for at least one of the segments $\left[s_{0}, z\right]$ and $\left[z, t_{0}\right]$, every point of $S$ sees this segment via $S$ : Otherwise, there would exist points $u, v \in S, p_{1}, p_{2} \in \operatorname{bdry} N \cap L_{1}$ and $p_{1}^{\prime}, p_{2}^{\prime} \in$ bdry $N \cap L_{2}$ such that the $\varepsilon$-segments at $z$ seen by both $u$ and $v$ would be either in the convex region bounded by $R\left(z, p_{1}\right) \cup R\left(z, p_{2}\right)$ or in the convex region bounded by $R\left(z, p_{1}^{\prime}\right) \cup R\left(z, p_{2}^{\prime}\right)$. This contradicts the fact that $u, v, w_{n}, y_{n}$ see a common $\varepsilon$-segment at $z$ for each value of $n$. We conclude that ker $S$ is a full 1-dimensional, and the proof for Case 1 is complete.

Case 2. Assume that $z \in$ bdry S. There are two possibilities to consider.

Case 2a. Suppose that there exist points $s, t, u$ in $S$ such that $z \in \operatorname{int} \operatorname{conv}\{s, t, u\}$. Then for two of these points, say $s$ and $t$, no point of $[s, z)$ sees any point of $[t, z)$ via $S$. Then $s$ and $t$ see a common $\varepsilon$-interval at $z$ in the closed region $R^{\prime}$ bounded by rays $R(t, z) \sim[t, z)$ and $R(s, z) \sim[s, z)$. We define $R$ to be that minimal sector of a circle containing all $\varepsilon$-intervals at $z$ seen by both $s$ and 
$t$. Then $R$ is bounded by segments $\left[z, s_{0}\right]$ and $\left[z, t_{0}\right]$ in $S$, and since $s, t, s_{0}, t_{0}$ see a common $\varepsilon$-interval at $z$ in $R$, certainly conv $\left\{s_{0}, z, t_{0}\right\} \subseteq S$. As before, order the points of bdry $R \sim\left(\left[z, s_{0}\right) \cup\left[z, t_{0}\right)\right)$ in a clockwise direction, and say $a<b$ on bdry $R \sim\left(\left[z, s_{0}\right) \cup\left[z, t_{0}\right)\right)$ if $a$ precedes $b$ in our clockwise ordering.

Assume that $s_{0}$ and $t_{0}$ are first and last points in our ordering. For each $y$ in $S$, define $D_{y}$ to be the convex hull of all $\varepsilon$-intervals at $z$ in $R$ seen by $y$, and let $s_{y}$ and $t_{y}$ be the first and last points of $D_{y}$ in bdry $R \sim\left(\left[z, s_{0}\right) \cup\left[z, t_{0}\right)\right)$. Clearly $s_{1} \equiv \operatorname{lub} s_{y} \leqq$ glb $t_{y} \equiv t_{1}$. Furthermore, a simple geometric argument reveals that every $y$ in $S$ sees the region conv $\left\{s_{0}, z, t_{0}\right\} \cap D_{y}$ via $S$. But $s_{0} \leqq s_{y} \leqq s_{1} \leqq t_{1} \leqq$ $t_{y} \leqq t_{0}$ on bdry $R$, so conv $\left\{s_{0}, z, t_{0}\right\} \cap \operatorname{conv}\left\{s_{1}, z, t_{1}\right\} \cong \operatorname{conv}\left\{s_{0}, z, t_{0}\right\} \cap D_{y}$, and $y$ sees conv $\left\{s_{0}, z, t_{0}\right\} \cap \operatorname{conv}\left\{s_{1}, z, t_{1}\right\}$ via $S$. This set is at least 1 -dimensional and so $\operatorname{dim} \operatorname{ker} S \geqq 1$, the required result.

Case 2b. Suppose that $z \in$ bdry conv $S$. Then there must exist a line $H$ supporting $S$ at $z$, with $S$ in the closed halfplane cl $H_{1}$ determined by $H$. Order the points $\left\{x: x \in \operatorname{cl} H_{1}\right.$ and $\left.\operatorname{dist}(z, x)=\varepsilon\right\}$ in a clockwise direction, and assume that $s_{0}$ and $t_{0}$ are the first and last points of $S$ in our ordering. Then conv $\left\{s_{0}, z, t_{0}\right\} \subseteq S$, since $s_{0}$ and $t_{0}$ see a common $\varepsilon$-interval at $z$.

If points $s_{0}, z, t_{0}$ are not collinear, then the argument in Case $2 \mathrm{a}$ above may be used to complete the proof. Hence consider the case in which $s_{0}, z, t_{0}$ lie in $H$. If $s_{0}=t_{0}$, the proof is trivial, so assume $s_{0}<z<t_{0}$. If $s_{0}$ and $t_{0}$ see a common interval at $z$ in $H_{1} \cup\{z\}$, then for some neighborhood $N$ of $z, N \cap S$ is convex, and the argument of Case 1 may be adapted to finish the proof. In case $s_{0}$ and $t_{0}$ see no such interval, then using the fact that every 4 points see a common $\varepsilon$-interval at $z$, it is easy to show that for at least one of the segments $\left[s_{0}, z\right]$ and $\left[t_{0}, z\right]$, every point of $S$ sees this segment via $S$. Hence we conclude that $\operatorname{dim} \operatorname{ker} S \geqq 1$ in Case 2 , and the proof of Theorem 1 is complete.

The following example illustrates that the number 4 in Theorem 1 is best possible.

ExAMPLE 1. Let $S$ be the set in Figure 1. Then every 3 points of $S$ see via $S$ at least one of the segments $\left[z, x_{i}\right], 1 \leqq i \leqq 4$, yet $\operatorname{ker} S=\{z\}$.

Example 2 shows that the uniform lower bound $\varepsilon$ on the segments seen by 4 points is necessary.

EXAMPLE 2. Let $S$ be the set in Figure 2. Then every 4 points see a common segment on the $x$-axis, but $\operatorname{ker} S$ is the origin. 


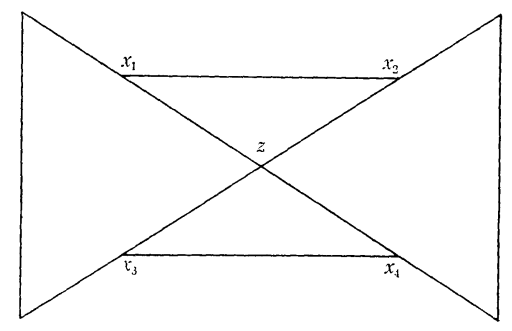

Figure 1

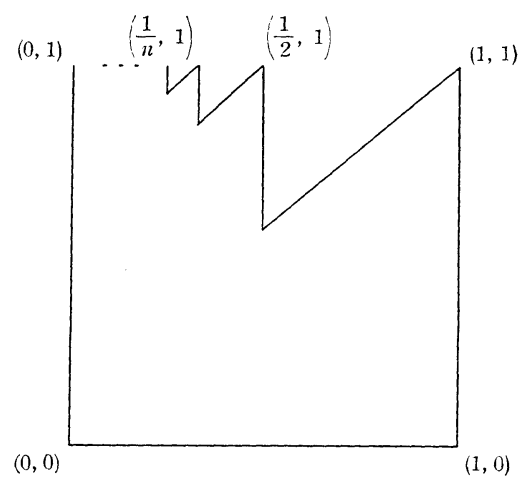

FIGURE 2

Our second theorem is not limited to the plane and is essentially a quantitative version of Krasnosel'skii's theorem.

THeORem 2. Let $S$ be a compact set in $R^{2}$. The dimension of ker $S$ is 2 if and only if for some $\varepsilon>0$, every 3 points of $S$ see via $S$ a common neighborhood of radius $\varepsilon$. The number 3 is best possible.

Proof. Again we need only establish the sufficiency of the condition. Clearly $S$ is starshaped, so select $z$ in ker $S$. We observe that for every 3 points $x_{1}, x_{2}, x_{3}$ in $S$, there corresponds a connected subset $T$ of $S$ such that dist $(z, t)=\varepsilon$ for each $t$ in $T$ and $\operatorname{conv}(T \cup\{z\})$ is a 2-dimensional subset of $S$. To verify this, let $N$ be a neighborhood of radius $\varepsilon$ seen by $x_{1}, x_{2}, x_{3}$. Then since $z \in \operatorname{ker} S$, $\operatorname{conv}\left(\left\{x_{i}, z\right\} \cup N\right) \subseteq S$ for each $i$, so $x_{i}$ sees conv $(\{z\} \cup N)$ via $S$. Letting $T=\{y: y \in$ $\operatorname{conv}(\{z\} \cup N)$, dist $(z, y)=\varepsilon\}, T$ satisfies the requirements given above.

Furthermore, letting $D$ denote the closed $\varepsilon$-disk about $z$, notice that $\operatorname{conv}(T \cup\{z\})$ is either $D$ or a nondegenerate sector of $D$. If we associate with each set $T$ the corresponding arc length $\delta(T)$ along bdry $D$, since $S$ is compact, the numbers $\delta(T)$ are bounded below by some positive number $\delta$. Therefore, for each $y \in S$, we may consider the collection $G_{y}$ of all sectors of $D$ seen by $y$ for which the corresponding arc length on $D$ is at least $\delta$. Then using the sets $G_{y}$, the 
argument in Theorem 1 may be appropriately modified and in fact simplified to complete the proof. The details are straightforward and hence are omitted.

To see that the number 3 of Theorem 2 is best possible, consider the following easy example.

EXAMPLE 3. Let $S$ be the set in Figure 3. Then every two points of $S$ see one of the regions $A_{i}$ via $S, 1 \leqq i \leqq 3$, yet $\operatorname{ker} S=\dot{\phi}$.

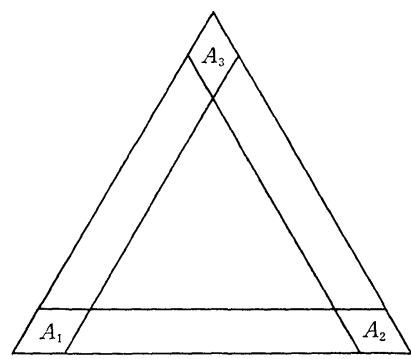

FiguRe 3

In conclusion, it is interesting to notice that both Theorems 1 and 2 fail completely and in fact no $f(k)$ is possible without the requirement that $S$ be compact.

EXAMPLE 4. To see that our set must be closed, let $S$ denote the unit disk with its center removed. Then every $j$-member subset of $S$ sees via $S$ an open sector having arc length $2 \pi / 2^{j}$, and every denumerable set of points sees a radius of $S$. Yet the set is not starshaped.

EXAMPle 5. To show that $S$ must be bounded, consider the following example by Hare and Kenelly [4]: Define $T_{n}=\{(x, y)$ :

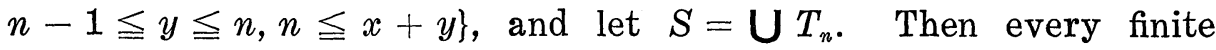
subset of $S$ sees via $S$ a common disk of radius $1 / 2$ in $T_{1}$, yet $S$ is not starshaped.

\section{REFERENCES}

1. Marilyn Breen, Sets in $R^{d}$ having (d-2)-dimensional kernels, Pacific J. Math., (to appear).

2. N. E. Foland and J. M. Marr, Sets with zero dimensional kernels, Pacific J. Math., 19 (1966), 429-432.

3. W. R. Hare, Jr. and J. W. Kenelly, Concerning sets with one point kernel, Nieuw Arch. Wisk., 14 (1966), 103-105.

4. - Intersections of maximal starshaped sets, Proc. Amer. Math. Soc., 19 (1968), 1299-1302. 
5. M. A. Krasnosel'skii, Sur un critère pour qu'un domaine soit étoile, Math. Sb., (61) 19 (1946), 309-310.

6. F. A. Toranzos, The dimension of the kernel of a starshaped set, Notices Amer. Math. Soc., 14 (1967), 832.

Received January 15, 1978.

The UNIVERSITY OF OKLAHOMA

NORMAN, OK 73019 



\section{PACIFIC JOURNAL OF MATHEMATICS}

\section{EDITORS}

DoNALD BABBITT (Managing Editor)

University of California

Los Angeles, California 90024

HUGo Rossi

University of Utah

Salt Lake City, UT 84112

C. C. MOORE and ANDREW OGG

University of California

Berkeley, CA 94720
J. DUGUNDJI

Department of Mathematics University of Southern Californı Los Angeles, California 90007

R. Finn and J. Milgram Stanford University Stanford, California 94305

\section{ASSOCIATE EDITORS}

E. F. BECKENBACH

B. H. Neumann
F. WOLF

K. YOSHIDA

\section{SUPPORTING INSTITUTIONS}

UNIVERSITY OF BRITISH COLUMBIA CALIFORNIA INSTITUTE OF TECHNOLOGY UNIVERSITY OF CALIFORNIA MONTANA STATE UNIVERSITY UNIVERSITY OF NEVADA, RENO NEW MEXICO STATE UNIVERSITY OREGON STATE UNIVERSITY UNIVERSITY OF OREGON
UNIVERSITY OF SOUTHERN CALIFORNIA STANFORD UNIVERSITY UNIVERSITY OF HAWAII UNIVERSITY OF TOKYO UNIVERSITY OF UTAH WASHINGTON STATE UNIVERSITY UNIVERSITY OF WASHINGTON 


\section{Pacific Journal of Mathematics}

Vol. 82, No. $1 \quad$ January, 1979

Werner Bäni, Subspaces of positive definite inner product spaces of countable dimension ...................................... 1

Marilyn Breen, The dimension of the kernel of a planar set..............

Kenneth Alfred Byrd, Right self-injective rings whose essential right ideals

are two-sided

Patrick Cousot and Radhia Cousot, Constructive versions of Tarski's fixed

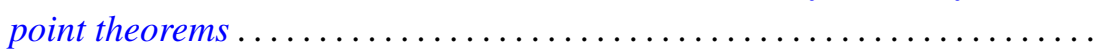

Ralph S. Freese, William A. Lampe and Walter Fuller Taylor, Congruence lattices of algebras of fixed similarity type. $I \ldots \ldots \ldots \ldots \ldots \ldots \ldots$

Cameron Gordon and Richard A. Litherland, On a theorem of Murasugi .....

Mauricio A. Gutiérrez, Concordance and homotopy. I. Fundamental

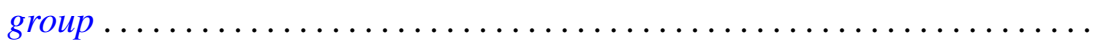

Richard I. Hartley, Metabelian representations of knot groups .............

Ted Hurley, Intersections of terms of polycentral series of free groups and free

Lie algebras ........................................

Roy Andrew Johnson, Some relationships between measures ............ 117

Oldřich Kowalski, On unitary automorphisms of solvable Lie algebras .......

Kee Yuen Lam, $K O$-equivalences and existence of nonsingular bilinear

maps...................................................

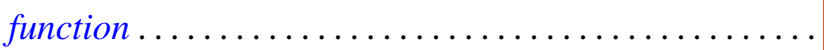

Robert A. Messer and Alden H. Wright, Embedding open 3-manifolds in compact 3-manifolds ............................

Gerald Ira Myerson, A combinatorial problem in finite fields. I . .

James Nelson, Jr. and Mohan S. Putcha, Word equations in a band of paths.

Baburao Govindrao Pachpatte and S. M. Singare, Discrete generalized Gronwall inequalities in three independent variables . .

William Lindall Paschke and Norberto Salinas, $C^{*}$-algebras associated with free products of groups ........................

Bruce Reznick, Banach spaces with polynomial norms ....

David Rusin, What is the probability that two elements of a finite group

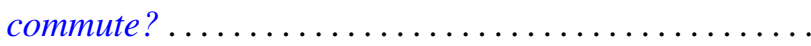

M. Shafii-Mousavi and Zbigniew Zielezny, On hypoelliptic differential operators of constant strength ...

Joseph Gail Stampfli, On selfadjoint derivation ranges .... . . .

Robert Charles Thompson, The case of equality in the matrix-valued triangle

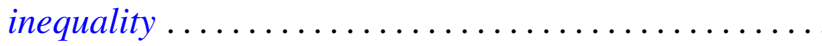

Marie Angela Vitulli, The obstruction of the formal moduli space in the negatively graded case. 